\title{
The cytosolic thiouridylase CTU2 of Arabidopsis thaliana is essential for posttranscriptional thiolation of tRNAs and influences root development
}

Matthias Philipp ${ }^{1}$, Florian John ${ }^{1,2}$ and Christoph Ringli ${ }^{1^{*}}$

\begin{abstract}
Background: A large number of post-transcriptional modifications of transfer RNAs (tRNAs) have been described in prokaryotes and eukaryotes. They are known to influence their stability, turnover, and chemical/physical properties. A specific subset of tRNAs contains a thiolated uridine residue at the wobble position to improve the codon-anticodon interaction and translational accuracy. The proteins involved in tRNA thiolation are reminiscent of prokaryotic sulfur transfer reactions and of the ubiquitylation process in eukaryotes. In plants, some of the proteins involved in this process have been identified and show a high degree of homology to their non-plant equivalents. For other proteins, the identification of the plant homologs is much less clear, due to the low conservation in protein sequence.

Results: This manuscript describes the identification of CTU2, the second CYTOPLASMIC THIOURIDYLASE protein of Arabidopsis thaliana. CTU2 is essential for tRNA thiolation and interacts with ROL5, the previously identified CTU1 homolog of Arabidopsis. CTU2 is ubiquitously expressed, yet its activity seems to be particularly important in root tissue. A ctu2 knock-out mutant shows an alteration in root development.

Conclusions: The analysis of CTU2 adds a new component to the so far characterized protein network involved in tRNA thiolation in Arabidopsis. CTU2 is essential for tRNA thiolation as a ctu2 mutant fails to perform this tRNA modification. The identified Arabidopsis CTU2 is the first CTU2-type protein from plants to be experimentally verified, which is important considering the limited conservation of these proteins between plant and non-plant species. Based on the Arabidopsis protein sequence, CTU2-type proteins of other plant species can now be readily identified.
\end{abstract}

Keywords: tRNA, thiolation, CYTOPLASMIC THIOURIDYLASE, CTU2, root, lateral roots, root hairs, LRX1, ROL5

\section{Background}

The accuracy of the translational machinery depends on the fidelity of codon recognition by the anticodons of transfer RNAs (tRNAs). tRNAs are short RNA molecules of 70-80 nucleotides that form defined secondary structures. Over one hundred different modifications of RNAs have been described, which are likely to influence their chemistry, metabolism, and stability [1]. In tRNAs, such modifications can influence the codon-anticodon complex formation. The uridine at the wobble base (U34) of tRNAs

\footnotetext{
* Correspondence: chringli@botinst.uzh.ch

'Institute of Plant Biology, University of Zürich, Zollikerstr 107, 8008 Zürich, Switzerland

Full list of author information is available at the end of the article
}

for Lys, Glu, and Gln is universally modified to 5-methyl2-thiouridine derivatives which enhance codon reading accuracy [2]. The process of U34 thiolation has been well studied in a variety of organisms and involves a number of proteins that activate and eventually transfer the sulfur onto the U34. In yeast, the E1 ligase-like protein Uba4p (MOCS3 in humans) activates and thiolates Urm1p (Urm1 in humans), a ubiquitin-related modifier (URM) protein, resulting in a thiocarboxylate at the C-terminal glycine. The sulfur is then further transferred via the activity of two CYTOPLASMIC THIOURIDYLASE proteins (CTUs), Ncs6p and Ncs2p (Ctu1 and Ctu2, respectively, in humans) to the uridine residue of the target tRNAs [3-6]). The two CTU proteins interact with each other 
and share common motifs, but only CTU1 is able to bind tRNAs. The ATP-binding motif PP-loop in these proteins suggests that the sulfur transfer is an energy-consuming reaction [7].

This process is reminiscent of the sulfur transfer reaction in prokaryotes [8] but also shares similarities with the eukaryotic ubiquitylation system. In yeast and human cell lines, the URM proteins have also been shown to be involved in protein conjugation, particularly under oxidative stress conditions. The biological significance of this process, however, remains to be determined $[6,9]$. The apparent conservation of the machinery involved in sulfur transfer and protein conjugation suggests an evolutionary relationship between these systems [10]. An additional, interesting aspect of loci involved in tRNA thiolation is that they frequently have been identified as modifiers of the TOR (Target of Rapamycin) network $[11,12]$, a major controller of cell growth in eukaryotes [13]. Since the regulation of translational activity is a target of the TOR pathway, it is quite possible that the lack of tRNA thiolation, which affects translational accuracy [2], has an impact on the TOR pathway via a feedback mechanism.

In plants, the investigation of the sulfur transfer reaction including thiolation of tRNAs is best investigated in Arabidopsis thaliana. The E1 ligase-like protein CNX5/ SIR1 was shown to be important for the biosynthesis of molybdopterin [14]. CNX5/SIR1 also transfers the sulfur to URM11 and URM12, the Arabidopsis homologs of URM proteins of yeast and humans [15,16]. ROL5 represents the CTU1 homolog of Arabidopsis which binds to URM11 and URM12 and transfers the sulfur group from the C-terminal thiocarboxylate of the URM proteins to the tRNAs. ROL5, URM11 and URM12 can complement corresponding yeast mutants and mutations in the Arabidopsis ROL5, URM11, and URM12 genes interfere with tRNA thiolation. These data show that URM11, URM12, and ROL5 code for the orthologs of the respective yeast proteins and that the sulfur transfer process is conserved in Arabidopsis [15-17]. The CTU2 ortholog, however, has so far not been identified.

In Arabidopsis, mutations in $c n x 5 /$ sir 1 severely impact plant growth, reflecting the position of this protein in several sulfur-dependent processes such as molybdopterin biosynthesis and tRNA thiolation $[14,15]$. In contrast, urm 11 urm 12 double and rol5 single mutants are mainly affected in root growth [16]. rol5 was initially identified as a suppressor of the root hair cell wall formation mutant lrx1 (leucine-rich repeat extensin 1) $[17,18]$. Correspondingly, mutating urm 11 and urm 12 also results in suppression of $\operatorname{lr} x 1$ [16]. A likely explanation for this observation is the impact of defective tRNA thiolation on the TOR network in Arabidopsis. Interfering with TOR signaling by RNAi constructs targeting the TOR kinase or using specific inhibitors of the TOR protein activity have similar effects on root development as found for the rols or urm11 urm $12 \mathrm{mu}$ tants [16,17,19-21].

This work experimentally identifies the second CYTOPLASMIC THIOURIDYLASE CTU2 of Arabidopsis. CTU2 is ubiquitously expressed in the plant and is essential for tRNA thiolation. Analysis of protein-protein interaction shows binding of CTU2 to ROL5, the CTU1 homolog of Arabidopsis. The analysis of a ctu2 knockout mutant reveals an effect on root development, suggesting that the modification of tRNAs is particularly important for root developmental processes.

\section{Results}

\section{CTU2 is poorly conserved among different species}

Based on protein homology, a potential CTU2 homolog of Arabidopsis was identified [TAIR, At4g35910]. An alignment of the proposed Arabidopsis CTU2 with those of distantly related plants such as potato (Solanum tuberosum) and rice (Oryza sativa) reveals $43 \%$ and 55\% identity, respectively, while the human protein shows around 20\% identical residues (Figure 1). Dewez et al. [7] identified a number of amino acid motifs that are conserved in CTU2-like proteins of a number of species and can thus be considered relevant for protein function. These sequences are not fully conserved in the plant CTU2 homologs and blocks of well conserved sequences among the plant proteins go beyond those identified as being important (Figure 1). The PP-loop motif (SGGKDS in CTU1-like proteins) involved in ATP binding $[4,7,22]$ is also found in CTU2-type proteins, but with the less conserved consensus sequence SGGXXS.

\section{The Arabidopsis CTU2 homolog is necessary for tRNA thiolation}

The moderate level of homology among CTU2 homologs made additional experiments necessary to provide evidence for the identified protein being the true Arabidopsis CTU2. Two ctu2 mutant alleles were identified in the publicly available seed stock, namely the Salk line 30197 and the Gabi-kat line 686B10-022973, which were named ctu2-1 and ctu2-2, respectively. Confirmation of the insertion sites in the CTU2 gene by determining the flanking sequence revealed that the T-DNA insertion in ctu2-1 is in the terminator sequence while the one in the ctu2-2 line is in the third exon, 879 bp downstream of the start codon (Figure 2A). To assess expression of the ctu 2 mutant alleles, mRNA was extracted from wildtype and the ctu 2 mutant plants and RT-PCR was performed. Only ctu2-2 revealed no gene expression while RNA from the $c t u 2-1$ allele still produced a PCR product (Figure 2B). Therefore, the $c t u 2-2$ allele was used for further experiments. 


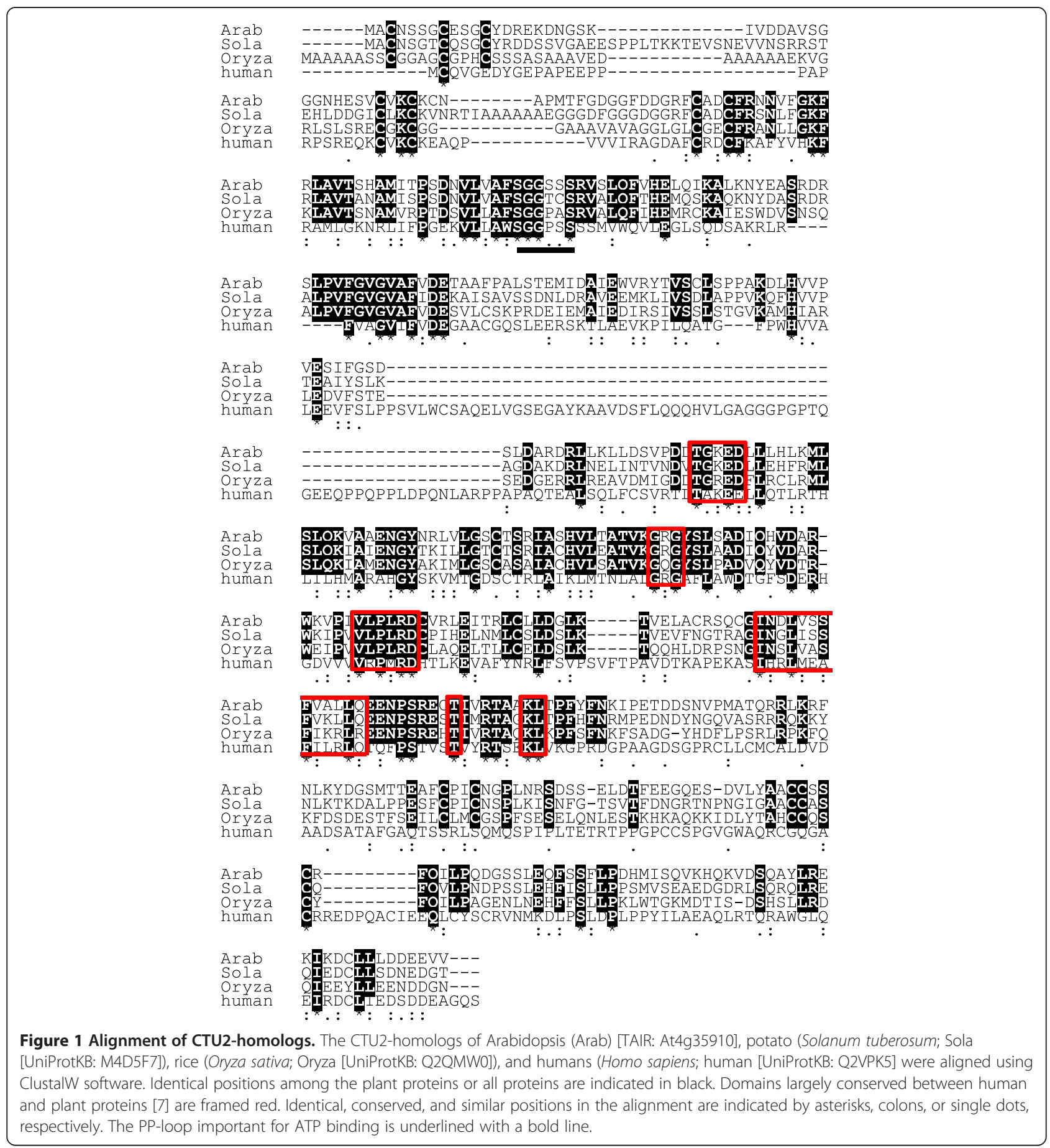

To analyze a possible effect of the $c t u 2-2$ mutation on tRNA thiolation, tRNA was isolated from wild-type and ctu2-2 mutant seedlings and separated on a polyacrylamide gel supplemented with $\mathrm{N}$-acryloylamino phenyl mercuric chloride (APM) which binds thiolated tRNAs, resulting in a higher molecular weight complex with slower migration in the gel. While tRNA isolated from the wild type showed the expected retarded band corresponding to thiolated tRNAs, these retarded tRNA species were absent in the ctu2-2 mutant (Figure 2C). Complementation of the ctu2-2 mutant with a wild-type CTU2 clone resulted in transgenic plants with reconstituted tRNA thiolation (Figure 2B, D). This suggests that the gene under investigation is indeed involved in tRNA thiolation.

CTU2 undergoes interaction with the CTU1-homolog ROL5 Different experimental evidence performed in several organisms established that the process of tRNA thiolation 


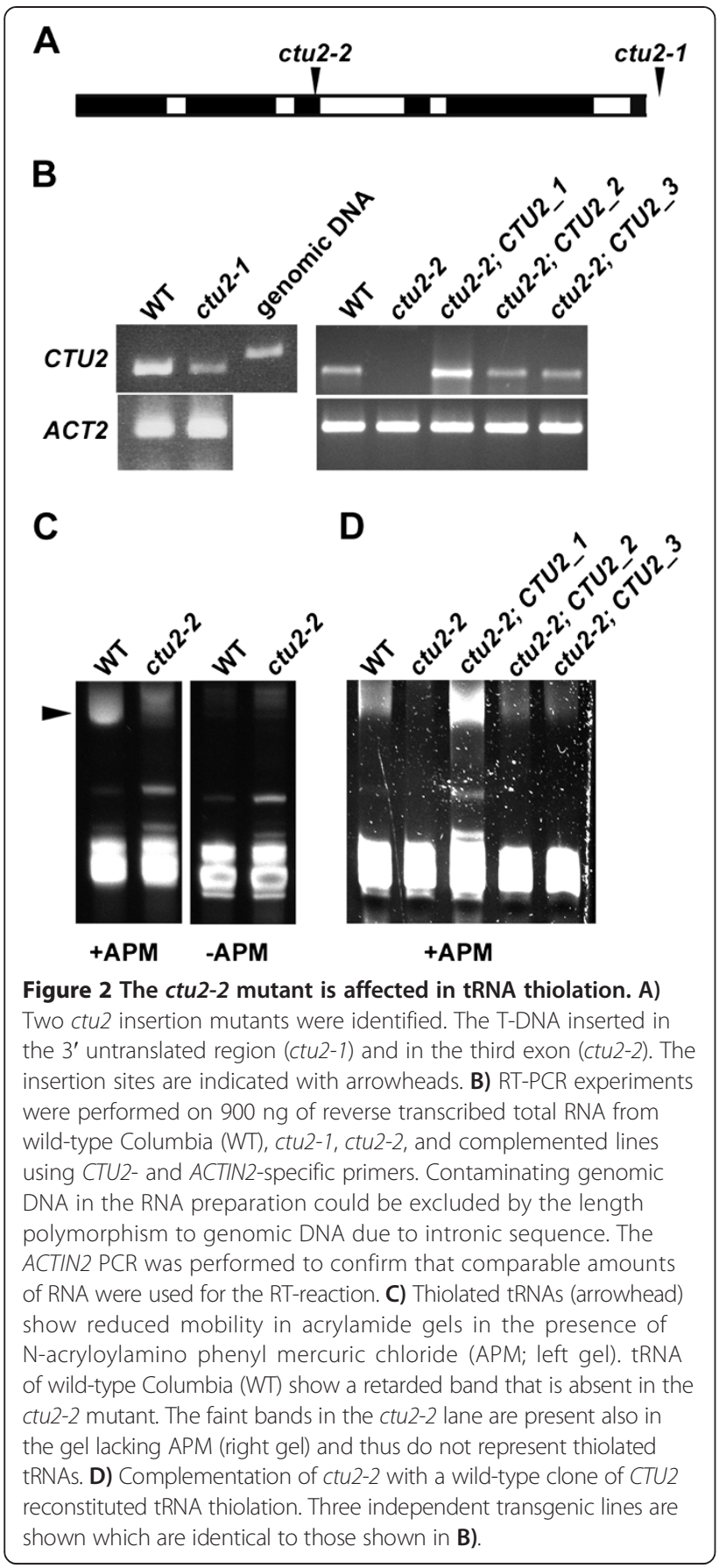

involves the interaction of CTU1-type and CTU2-type proteins $[4,5,7]$. The CTU1-type protein is essential for tRNA thiolation and is encoded in Arabidopsis by ROL5 [17]. To provide further evidence that the identified Arabidopsis protein is indeed CTU2, the interaction with ROL5 was tested in a yeast-two-hybrid experiment. Transformation of yeast cells with ROL5 and CTU2 cDNAs in bait and prey vectors, respectively, revealed growth of yeast cells on appropriate selective media indicative of the interaction of the two proteins. In addition, strong $\beta$-galactosidase activity was observed, confirming the activation of the reporter system in yeast. In control experiments with the vectors containing CTU2 or ROL5 and the second empty plasmid did not show any growth of yeast under selective conditions, excluding autoactivation by CTU2 or ROL5 alone (Figure 3). Hence, ROL5 and the proposed CTU2 undergo protein-protein interaction, as is expected for the CTU1- and CTU2homologs of Arabidopsis.

\section{CTU2 is ubiquitously expressed and influences root architecture}

To investigate the expression pattern of CTU2, a promoter:GUS fusion construct was produced and transformed into wild-type Arabidopsis. GUS activity was monitored in several independent transgenic lines. At the seedlings stage, GUS activity leading to blue staining was observed in all tissues, i.e. roots, hypocotyl, rosette leaves, and cotyledons. Also in adult plants, GUS staining was found in all tissues (Figure 4). Hence, CTU2 seems expressed evenly in all tissues, which is in agreement with microarray data [23,24].

To investigate the importance of CTU2 in plant development, the ctu2-2 mutant was analyzed for aberrant morphological phenotypes. Seedlings grown for 8 days on MS agar plates in a vertical orientation revealed a reduction in lateral root formation and, hence, lateral root density in the mutant compared to the wild type (Figure 5A). Mutant lines complemented with the wild-type CTU2

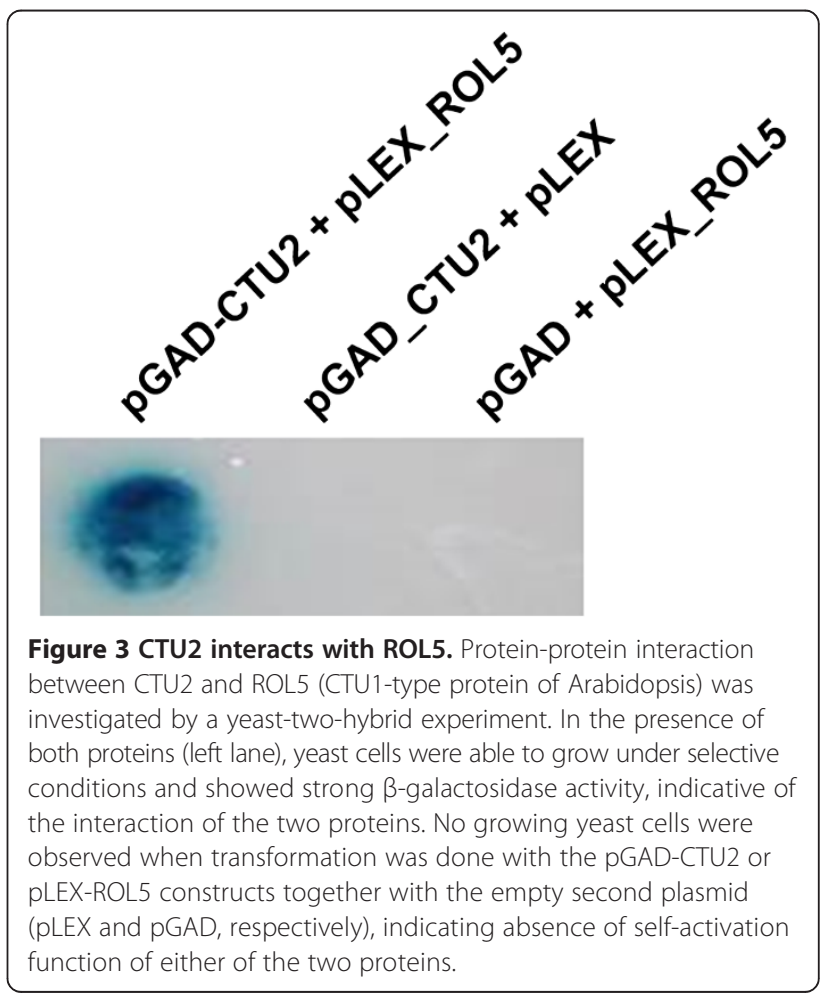




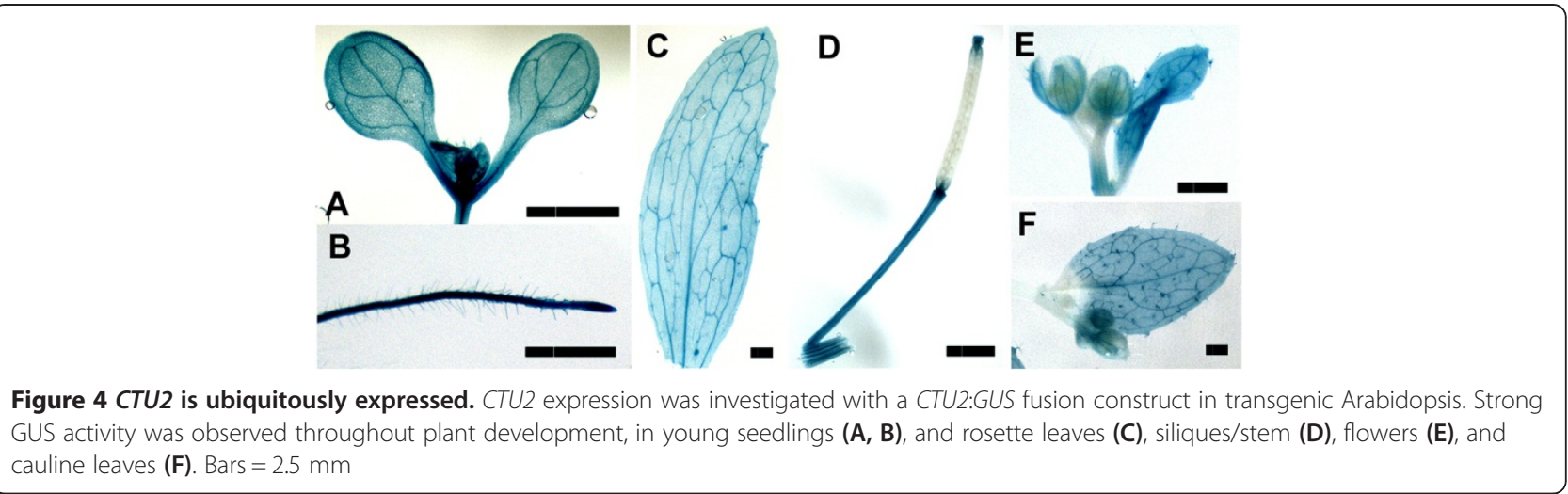

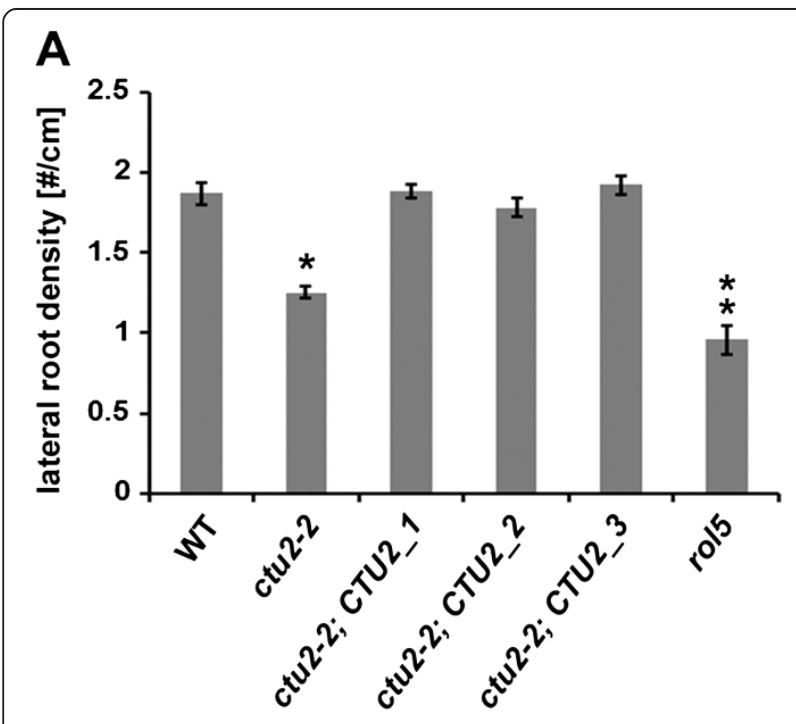

B

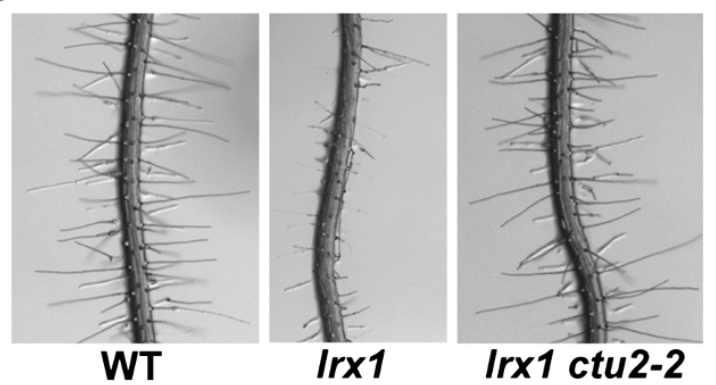

Figure 5 The ctu2-2 mutant is affected in root development. A) The ctu2-2 mutant shows a reduction in lateral root formation compared to wild-type Columbia (WT) and the complemented ctu2-2 mutants (indicated by an asterisk; $p=0.01 ; n \geq 15$ ), determined after 8 days of growth in a vertical orientation. The complementation lines are the same as shown in Figure 2. The tRNA thiolation-defective rol5 mutant also shows a reduction, which is significantly stronger than in ctu2-2 (indicated by two asterisks; $p=0.05 ; n \geq 15$ ). Error bars represent the standard error of the mean. B) In contrast to wild-type Columbia, 7 days-old Irx 1 mutant seedlings develop aberrant root hairs. This phenotype is suppressed by ctu2-2, resulting in wild type-like root hair formation in the Irx1 ctu2-2 double mutant. construct showed reversion to wild type-like lateral root formation, confirming that the ctu2-2 mutation is causing this developmental defect. Since the same effect on lateral root development was observed in the tRNA thiolationdeficient urm11 urm12 double mutant [16], the tRNA thiolation-deficient rol5 mutant was also analyzed. The quantification of lateral root formation revealed a reduction in the lateral root density in the rol5 mutant that was even stronger than in the ctu2-2 line (Figure 5A). The similar effect of the different mutations blocking tRNA thiolation suggests that root development is particularly sensitive to changes in this type of tRNA modification.

The rol5 mutant was initially identified as a suppressor of the root hair formation mutant $\operatorname{lr} x 1[17,18]$. To test whether $c t u 2-2$ would have the same effect on $l r x 1$, an Irx1 ctu2-2 double mutant was produced. While wildtype seedlings developed normal root hairs, those of $l r x 1$ mutant seedlings showed the previously reported deformation phenotype $[17,18]$. The lrx 1 ctu2-2 double mutant seedlings, however, developed wild type-like root hairs (Figure 5B). The phenotypic penetrance in the different lines was very high and consistent. Hence, also the $c t u 2-2$ mutation acts as a suppressor of $\operatorname{lrx} 1$.

\section{Discussion}

tRNAs of bacteria and eukaryotes are altered by a variety of modifications [25]. The uridine in the wobble position of tRNAs recognizing the split-codon boxes of Lys, Glu, and Gln codons are universally modified to 5-methyl2-thiouridine derivatives $\left(\mathrm{xm}^{5} \mathrm{~s}^{2} \mathrm{U}\right)$; the cytosolic tRNAs of eukaryotes containing 5-methoxycarbonylmethyl-2-thiouridine $\left(\mathrm{mcm}^{5} \mathrm{~s}^{2} \mathrm{U}\right)$ [26]. The thiolation process requires a number of proteins that were identified in diverse organisms such as yeast, $C$. elegans, and humans. Among these proteins are an E1-like ligase, a ubiquitin-related modifier and two cytoplasmic thiouridylase (CTU) proteins [3-5,7]. This work describes the experimental identification of the second CYTOPLASMIC THIOURIDYLASE, CTU2. While other plant homologs of the proteins involved could be readily identified based on amino acid sequence 
homology, [14-17], the level of homology of CTU2-type proteins across eukaryotes is rather low, making experimental evidence for the correct annotation of the Arabidopsis homolog necessary. The proteins of Arabidopsis and yeast or humans only share $20 \%$ identity, which is drastically lower than between CTU1-type proteins. These proteins in yeast and Arabidopsis (Ncs6p and ROL5, respectively) share $54 \%$ identical residues [17]. This difference in conservation of the protein sequence suggests that CTU2-type proteins have undergone a stronger divergent evolution than CTU1-type proteins. A low sequence homology can indicate that initial substitutions of amino acids had to be compensated for by secondary mutations to maintain protein function, resulting in constant conformational characteristics despite low sequence homology $[27,28]$. Alternatively, the function of CTU2-type proteins might tolerate more changes in the protein without affecting its intrinsic activity. Despite the limited sequence homology, the identified protein is highly likely to be CTU2 since it is essential for tRNA thiolation and interacts with the CTU1-type protein ROL5. The interaction of CTU1- and CTU2-type proteins has been wellestablished in several species $[4,5]$. In in vitro experiments, the CTU1-CTU2 complex has been shown to be sufficient for tRNA thiolation, even though at a very low level [7]. In vivo, however, the accumulation of biologically significant amounts of thiolated tRNAs requires the other protein components of this pathway.

CTU2 appears to be expressed in most if not all tissues. This is supported by the staining of transgenic plants containing an CTU2:GUS fusion construct and by gene expression profile analysis platforms such as Genevestigator [23] or At GenExpress Visualization Tool [24] that both indicate a moderate expression in all tissues. Despite the ubiquitous occurrence of tRNA thiolation, an obvious mutant phenotype of ctu2-2 is limited to root tissue. This might be related to the laboratory conditions under which the plants were grown, or the increased susceptibility of this tissue to changes in tRNA modification.

\section{The impact of tRNA thiolation on developmental processes}

Thiolation of the wobble position of specific tRNAs is important for effective translation by restricting basepairing capability, preventing misreading of other nearcognate codons, and influencing the thermostability of the codon-anticodon interaction [29-31]. Yeast and worm mutants defective in this tRNA modification exhibit a temperature-sensitive growth phenotype [7]. By contrast, increased CTU1 activity parallels increased cell growth, as observed in certain types of human cancer [32]. Hence, the level of tRNA thiolation can have a substantial effect on cell viability.
In yeast, blocking tRNA thiolation by mutating Ncs6, Ncs2, or Urm1 causes a change in growth properties, resulting in the failure of yeast cells to induce pseudohyphal growth, an otherwise common response to nutrient-limited conditions in order to improve nutrient uptake efficiency $[12,33]$. In plants, the tRNA thiolation mutants ctu2-2, urm11 urm12, and rol5 exhibit a reduction in lateral root density and are also affected in root hair development, exemplified by the suppression of the $\operatorname{lr} x 1$ root hair phenotype in $l r x 1$ ctu2-2, lrx 1 urm11 urm12, and lrx 1 rol5 mutants $([16,17]$, this work). Similar to pseudohyphae in yeast, the plant root system is important for nutrient uptake, indicating that changes in tRNA thiolation affect functionally related differentiation processes in very distinct organisms. Rather than being a coincident, this similarity might have a common basis in the TOR pathway, which senses nutrient availability and growth factors and adapts cell growth to prevailing conditions via influencing e.g. translation, mitochondrial activity, or cytoskeleton dynamics [34]. The lack of tRNA thiolation affects translational activity, which is likely to feedback to the TOR network [11,12], resulting in developmental alterations. Indeed, inhibiting TOR signaling by different means in Arabidopsis results in reduced root development and suppression of the $l r x 1$ root hair phenotype as found for rol5, urm11 urm12, and ctu2-2 [17,19-21,35].

\section{Conclusions}

This work describes the experimental validation of the CTU2 protein of Arabidopsis thaliana, the second CYTOPLASMIC THIOURIDYLASE. The finding that protein-protein interaction between CTU1- and CTU2type proteins of Arabidopsis takes place and that the CTU2 protein is essential for tRNA thiolation provides further support that the protein machinery involved in tRNA thiolation is well conserved among eukaryotes. The thiolation of tRNAs is not only physiologically important but is also connected to cell growth control. Hence, in future experiments it will be interesting to assess the potential of modifying plant growth properties and stress responses via modulating expression levels of genes involved in tRNA modification.

\section{Methods}

Plant material, growth conditions, and molecular markers

All Arabidopsis lines used are in the Columbia background. The lrxi-1 and rol5-1 alleles used in this study were previously described $[17,36]$. The $c t u 2-1$ and the ctu2-2 allele are the T-DNA insertion lines Salk_30197 and the Gabi-kat line 686B10-022973, respectively. Seed sterilization and plant growth was done as described [37].

For selection of mutant plants, the lrx1-1 allele was detected by a marker described in Diet et al. [36]. The T-DNA insertion in ctu2-1 was detected with the gene- 
specific primer CTU2_F.1 TATGATGGATCAATGAC TACTGAAG and the T-DNA specific primer $S A L K L L b$ GCGTGGACCGCTTGCTGCAACT. For $c t u 2-2$, the genespecific primer CTU2_1050F CTCGTGTTTGTCTCCACC TGCTAA and the T-DNA specific primer Gabi_LB CCC ATTTGGACGTGAATGTAGACAC were used. The CTU2 wild-type copy in ctu2-1 was amplified with the primers CTU2_F.1 TATGATGGATCAATGACTACTGAAG and CTU2_R.1 GTAGATGCTTCATTCAATTGCTC; the one of $c t u 2-2$ was amplified with the primers CTU2_1050F CTCGTGTTTGTCTCCACCTGCTAA and CTU2_1777R CTGCCCTGCCCAGAATATGTGACG.

\section{DNA constructs}

For complementation of the ctu2-2 allele, the genomic clone of CTU2 including $2.1 \mathrm{~kb}$ of promoter and $0.75 \mathrm{~kb}$ of terminator sequence was amplified from wild-type Columbia genomic DNA with the primers CTU2_promF TGGCATACCGACTTACTAGCTTG and CTU2_TermR TCTCACCATTCTAAAGCTTTGATC and cloned in pGEM-T easy (Promega). The insert of a correct clone was cut out with NotI and cloned into the plant transformation vector pBART [38] which is identical to pART27 [39] but contains a basta- instead of kanamycin-resistance gene for plant selection. For the CTU2:GUS fusion construct, the CTU2 promoter was cloned into the plant transformation vector pGPTV-Bar [40].

For the yeast-two-hybrid experiment, a CTU2 cDNA was amplified from Columbia with the primers XbaI_At4g 35910_1F TCTAGAATGGCTTGTAATTCCTCAGG and BamHI_At4g35910_1R GGATCCTTAGACAACCTCTTC ATCGT and cloned into pGAD-HA cut with $X b a \mathrm{I}$ and $B a m H I$. The ROL5 cDNA clone was amplified from a previously amplified cDNA clone [17] with the primers KpnI_At2g44270_1F GGTACCATGGAGGCCAAGAA CAAGAAAGCAG and SmaI_At2g44270_1R CCCGGG TTAGAAATCCAGAGATCCACATTG and inserted in pLEXA_N (Dualsystems) cut with KpnI and SmaI.

\section{Plant transformation and GUS staining}

Plant transformation was performed as described [41], and transgenic seedlings were selected using $20 \mathrm{mg} / \mathrm{L}$ Basta and propagated to the next generation. GUS staining was performed in the T2 generation of five independent transgenic lines, in $50 \mathrm{mM}$ Na-phosphate $\mathrm{pH}$ 7.0, $10 \mathrm{mM}$ EDTA, $0.1 \%$ Triton X-100, and $1 \mathrm{mM}$ 5-bromo4-chloro-3-indolyl- $\beta$-D-glucuronic acid during $4 \mathrm{hrs}$ at $37^{\circ} \mathrm{C}$.

\section{tRNA extraction and analysis}

Arabidopsis seedlings were grown vertically on half-strength MS plates for 14 days as described [37]. Approximately 250 seedlings were used per extraction. The seedlings were grinded in liquid nitrogen and the material was extracted two times with $8 \mathrm{~mL}$ acidic phenol (Sigma), $0.8 \mathrm{~mL}$ chloroform and once with $4 \mathrm{~mL}$ acidic phenol, 0.4 mL chloroform. After extraction, tRNA was purified with AX100 columns from MACHEREY NAGEL following the manufacturer's instructions. For analysis, the purified tRNA was separated on an acrylamide gel supplemented with $\mathrm{N}$-acryloylamino phenyl mercuric chloride (APM) by the method adapted from Björk et al. [2].

\section{RT-PCR}

Wild-type Columbia and ctu2-2 mutant seedlings were grown as descried [37] for 7 days in a vertical orientation. Entire seedlings were frozen in liquid nitrogen, grinded, and total RNA was extracted using the SV Total RNA Isolation System kit (Promega). The reverse transcription was conducted with $900 \mathrm{ng}$ of total RNA using the i_script kit (Biorad). One tenth of the volume of the cDNA synthesis reaction was then used for RTPCR using the primer pairs ACTIN2F AATGAGCTTCG TATTGCTCC and ACTIN2R GCACAGTGTGAGACA CACC, and CTU2_rt_for CTCGTGTTTGTCTCCACC TGCTAA and CTU2_rt_rev TAGACAACCTCTTCATC GTCCAAG. The ACTIN2 PCR was done with 25 cycles, the CTU2 PCRs with 34 cycles of amplification.

\section{Root phenotype analysis}

Phenotypic observations and GUS activity analysis were done with a Leica LZ M125 stereomicroscope. Data points of lateral root development were taken after 8 days of growth in a vertical orientation. A t-test was performed to assess statistical significance of differences in lateral root formation. For the root hair phenotype, over 30 seedlings of each line were analyzed. Phenotypic variation was very small, i.e. each genotype showed a highly consistent root hair phenotype.

\section{Yeast strains and growth conditions}

Transformation and growth of yeast for the yeast-twohybrid experiment was done following manufacturer's instructions (Dualsystems).

\section{Accession numbers}

The accession numbers of the genes used in this study are as follows: CTU2: At4g35910; LRX1: At1g12040; ROL5: At2g44270.

Competing interests

The authors declare that they have no competing interests.

Author's contributions

MP and FJ have made substantial contributions to the acquisition of the data and have been involved in writing the manuscript. CR has designed the project, contributed to data acquisition and written the manuscript. All authors read and approved the final manuscript. 


\section{Acknowledgements}

We are grateful to Anja Meury Bechtel for excellent technical assistance and to Dr. S. Leidel for providing APM. This work was supported by the Swiss National Science Foundation grants Nr. 122157 and 138472.

\section{Author details}

${ }^{1}$ Institute of Plant Biology, University of Zürich, Zollikerstr 107, 8008 Zürich, Switzerland. ${ }^{2}$ Current Address: Thermo Scientific, Wohlen, Switzerland.

\section{Received: 18 October 2013 Accepted: 22 April 2014}

Published: 28 April 2014

\section{References}

1. Rozenski J, Crain PF, McCloskey JA: The RNA modification database: 1999 update. Nucleic Acids Res 1999, 27(1):196-197.

2. Björk GR, Huang B, Persson OP, Byström AS: A conserved modified wobble nucleoside ( $\mathrm{mcm} 5 \mathrm{~s} 2 \mathrm{U})$ in lysyl-tRNA is required for viability in yeast. RNA 2007, 13(8):1245-1255.

3. Schlieker CD, Van der Veen AG, Damon JR, Spooner E, Ploegh HL: A functional proteomics approach links the ubiquitin-related modifier Urm1 to a tRNA modification pathway. Proc Natl Acad Sci U S A 2008, 105(47):18255-18260.

4. Leidel S, Pedrioli PG, Bucher T, Brost R, Costanzo M, Schmidt A, Aebersold R, Boone C, Hofmann K, Peter M: Ubiquitin-related modifier Urm1 acts as a sulphur carrier in thiolation of eukaryotic transfer RNA. Nature 2009, 458(7235):228-232.

5. Noma A, Sakaguchi Y, Suzuki T: Mechanistic characterization of the sulfur-relay system for eukaryotic 2-thiouridine biogenesis at tRNA wobble positions. Nucleic Acids Res 2009, 37(4):1335-1352.

6. Van der Veen AG, Schorpp K, Schlieker C, Buti L, Damon JR, Spooner E, Ploegh $\mathrm{HL}$, Jentsch S: Role of the ubiquitin-like protein Urm1 as a noncanonical lysine-directed protein modifier. Proc Natl Acad Sci U S A 2011, 108(5):1763-1770

7. Dewez M, Bauer F, Dieu M, Raes M, Vandenhaute J, Hermand D: The conserved Wobble uridine tRNA thiolase Ctu1-Ctu2 is required to maintain genome integrity. Proc Natl Acad Sci U S A 2008, 105(14):5459-5464.

8. Miranda HV, Nembhard N, Su D, Hepowit N, Krause DJ, Pritz JR, Phillips C, Söll D, Maupin-Furlow JA: E1- and ubiquitin-like proteins provide a direct link between protein conjugation and sulfur transfer in archaea. Proc Natl Acad Sci U S A 2011, 108(11):4417-4422.

9. Goehring AS, Rivers DM, Sprague GF: Urmylation: a ubiquitin-like pathway that functions during invasive growth and budding in yeast. Mol Bio/ Cell 2003, 14(11):4329-4341.

10. Petroski MD, Salvesen GS, Wolf DA: Urm1 couples sulfur transfer to ubiquitin-like protein function in oxidative stress. Proc Natl Acad Sci U S A 2011, 108(5):1749-1750.

11. Chan TF, Carvalho J, Riles L, Zheng XFS: A chemical genomics approach toward understanding the global functions of the target of rapamycin protein (TOR). Proc Natl Acad Sci U S A 2000, 97(24):13227-13232

12. Goehring AS, Rivers DM, Sprague GF: Attachment of the ubiquitin-related protein Urm1p to the antioxidant protein Ahp1p. Eukaryot Cell 2003, 2(5):930-936.

13. Laplante M, Sabatini DM: mTOR signaling in growth control and disease. Cell 2012, 149(2):274-293.

14. Teschner J, Lachmann N, Schulze J, Geisler M, Selbach K, Santamaria-Araujo J, Balk J, Mendel RR, Bittner F: A novel role for Arabidopsis mitochondrial ABC transporter ATM3 in molybdenum cofactor biosynthesis. Plant Cell 2010, 22(2):468-480

15. Nakai Y, Harada A, Hashiguchi Y, Nakai M, Hayashi H: Arabidopsis molybdopterin biosynthesis protein $\mathrm{CNX} 5$ collaborates with the ubiquitin-like protein URM11 in the thio-modification of tRNA. J Biol Chem 2012, 287(36):30874-30884

16. John F, Philipp M, Leiber RM, Errafi S, Ringli C: Ubiquitin-Related modifiers of Arabidopsis thaliana influence root development. PLoS One 2014, 9(1):22.

17. Leiber RM, John F, Verhertbruggen Y, Diet A, Knox JP, Ringli C: The TOR pathway modulates the structure of cell walls in Arabidopsis. Plant Cell 2010, 22(6):1898-1908.

18. Baumberger N, Ringli C, Keller B: The chimeric leucine-rich repeat/extensin cell wall protein LRX1 is required for root hair morphogenesis in Arabidopsis thaliana. Genes Dev 2001, 15(9):1128-1139.

19. Deprost D, Yao L, Sormani R, Moreau M, Leterreux G, Nicolai M, Bedu M, Robaglia C, Meyer C: The Arabidopsis TOR kinase links plant growth, yield, stress resistance and mRNA translation. EMBO Rep 2007, 8(9):864-870.
20. Ren $M Z$, Venglat $P$, Qiu SQ, Feng $L$, Cao YG, Wang E, Xiang DQ, Wang JH, Alexander D, Chalivendra S, David L, Autar M, Gopalan S, Raju D: Target of Rapamycin signaling regulates metabolism, growth, and life span in Arabidopsis. Plant Cell 2012, 24(12):4850-4874.

21. Xiong Y, Sheen J: Rapamycin and glucose-target of rapamycin (TOR) protein signaling in plants. J Biol Chem 2012, 287(4):2836-2842.

22. Bork P, Koonin EV: A P-loop-like motif in a widespread ATP pyrophosphatase domain: implications for the evolution of sequence motifs and enzyme activity. Proteins 1994, 20(4):347-355.

23. Zimmermann P, Hirsch-Hoffmann M, Hennig L, Gruissem W: GENEVESTIGATOR: Arabidopsis microarray database and analysis toolbox. Plant Physiol 2004, 136(1):2621-2632.

24. Schmid M, Davison TS, Henz SR, Pape UJ, Demar M, Vingron M, Scholkopf B, Weigel D, Lohmann JU: A gene expression map of Arabidopsis thaliana development. Nat Genet 2005, 37(5):501-506.

25. Phizicky EM, Hopper AK: tRNA biology charges to the front. Genes Dev 2010, 24(17):1832-1860.

26. Suzuki T: Biosynthesis and function of tRNA wobble modifications. In Topics in Current Genetics. Edited by Grosjean H.; 2005:23-69. vol. 12.

27. Flores TP, Orengo CA, Moss DS, Thornton JM: Comparison of conformational characteristics in structurally similar protein pairs. Protein Sci 1993, 2(11):1811-1826.

28. Williams SG, Lovell SC: The effect of sequence evolution on protein structural divergence. Mol Biol Evol 2009, 26(5):1055-1065.

29. Kruger MK, Pedersen S, Hagervall TG, Sorensen MA: The modification of the wobble base of tRNA (Glu) modulates the translation rate of glutamic acid codons in vivo. J Mol Biol 1998, 284(3):621-631.

30. Yarian C, Townsend H, Czestkowski W, Sochacka E, Malkiewicz AJ, Guenther R, Miskiewicz A, Agris PF: Accurate translation of the genetic code depends on tRNA modified nucleosides. J Biol Chem 2002, 277(19):16391-16395.

31. Murphy FV, Ramakrishnan V, Malkiewicz A, Agris PF: The role of modifications in codon discrimination by tRNA (Lys) UUU. Nat Struct Mol Biol 2004, 11(12):1186-1191.

32. Yousef GM, Borgono CA, Michael IP, Davidian C, Stephan C, Jung K, Diamandis EP: Molecular cloning of a new gene which is differentially expressed in breast and prostate cancers. Tumour Biol 2004, 25(3):122-133

33. Gimeno CJ, Ljungdahl PO, Styles CA, Fink GR: Unipolar cell divisions in the yeast Saccharomyces cerevisiae lead to filamentous growth-regulation by starvation and RAS. Cell 1992, 68(6):1077-1090.

34. Wullschleger $S$, Loewith $R$, Hall MN: TOR signaling in growth and metabolism. Cell 2006, 127(3):5-19.

35. Sormani R, Yao L, Menand B, Ennar N, Lecampion C, Meyer C, Robaglia C: Saccharomyces cerevisiae FKBP12 binds Arabidopsis thaliana TOR and its expression in plants leads to rapamycin susceptibility. BMC Plant Biol 2007, 7:26.

36. Diet $A$, Brunner $S$, Ringli $C:$ The enl mutants enhance the Irx 1 root hair mutant phenotype of Arabidopsis thaliana. Plant Cell Physiol 2004, 45:734-741.

37. Kuhn BM, Geisler M, Bigler L, Ringli C: Flavonols accumulate asymmetrically and affect auxin transport in Arabidopsis. Plant Physiol 2011, 156(2):585-595.

38. Stintzi A, Browse J: The Arabidopsis male-sterile mutant, opr3, lacks the 12-oxophytodienoic acid reductase required for jasmonate synthesis. Proc Natl Acad Sci U S A 2000, 97(19):10625-10630.

39. Gleave AP: A versatile binary vector system with a T-DNA organisational structure conducive to efficient integration of cloned DNA into the plant genome. Plant Mol Biol 1992, 20(6):1203-1207.

40. Becker D, Kemper E, Schell J, Masterson R: New plant binary vectors with selectable markers located proximal to the left T-DNA border. Plant Mol Biol 1992, 20(6):1195-1197.

41. Diet A, Link B, Seifert GJ, Schellenberg B, Wagner U, Pauly M, Reiter WD, Ringli C: The Arabidopsis root hair cell wall formation mutant Irx 1 is suppressed by mutations in the RHM1 gene encoding a UDP-L-rhamnose synthase. Plant Cell 2006, 18(7):1630-1641.

doi:10.1186/1471-2229-14-109

Cite this article as: Philipp et al.: The cytosolic thiouridylase CTU2 of Arabidopsis thaliana is essential for posttranscriptional thiolation of tRNAs and influences root development. BMC Plant Biology 2014 14:109. 